\title{
Influential Article Review - An Analytic View to Government Spending Strategy Response Guidelines
}

\author{
Dominika Roy \\ Cassius Buxton
}

Simra Lawson

This paper examines governance. We present insights from a highly influential paper. Here are the highlights from this paper: We present an algorithmic approach for the design of fiscal policy rules. In particular, using algorithmic feedback control techniques, we design linear feedback policy rules such that predetermined target levels for GDP and public debt are simultaneously, exactly tracked. We run a number of simulations in order to examine the effects of different policy response rates and the overall effectiveness of the proposed methodology. For our overseas readers, we then present the insights from this paper in Spanish, French, Portuguese, and German.

Keywords: fiscal policy, public debt, linear feedback control, algorithmic control

\section{SUMMARY}

- As already stated in the introduction, the model we chose is a linear, deterministic variant of the multiplier-accelerator model coupled with the government budget constraint. This model has been extensively used in the relevant literature, due to its tractability and because it can easily be extended to both nonlinear and stochastic variants.

- Our aim is to design linear feedback policy rules for short-term fiscal policy interventions.

- In order to design the feedback policy rules, we use a technique from the control theory literature known as model matching; it is a completely parameterized technique, allowing for proper symbolic algorithms to be developed.

- From an economic policy point of view, the timing of the policy action is a central issue. In particular, given the lags associated with policy conduct, should the government immediately react to signs of a downturn in economic activity via, for example, a frontloaded disbursement of investment funds or would it be preferable to adopt a more gradual response? Moreover, how do the lags associated with policy conduct affect the actual implementation of the policy and its effects? In the analysis presented in Sect.

- We assume that the government aims for a $1 \%$ per period increase in GDP levels and a corresponding decrease in the levels of debt. 
- The numerical simulations indicate that the faster the response of the government in the face of a downturn, the smaller the necessary changes in the composition of government expenditure. On the contrary, if the response of the government is delayed, then large and abrupt changes are required rendering the policy plans politically infeasible.

- As we can clearly see in the figures, the response time is critical regarding the composition of total government expenditures, i.e., the allocation between investment-related expenditures G I and general government expenditures $\mathrm{G}$ w as well as regarding the necessary changes in the size of the policy instruments.

\section{HIGHLY INFLUENTIAL ARTICLE}

We used the following article as a basis of our evaluation:

Kostarakos, I., \& Kotsios, S. (2017). Feedback policy rules for government spending: An algorithmic approach. Journal of Economic Structures, 6(1). https://doi.org/10.1186/s40008-017-0065-z

This is the link to the publisher's website:

https://journalofeconomicstructures.springeropen.com/articles/10.1186/s40008-017-0065-z

\section{INTRODUCTION}

One of the most important objectives of economic policy is to ensure, via the appropriate manipulation of the available policy instruments (control variables), that the economic system tracks, as closely as possible, a desired path for the policy targets (outputs). One of the approaches that has been utilized for the design of economic policy is the feedback approach, stemming from the mathematical control theory literature. Various aspects of the feedback methodology have been utilized for the purposes of policy design for more than 50 years, starting with the use of PID controllers in the seminal paper by Phillips (1954). These aspects range from (stochastic) optimal feedback control (see, among others, Amman and Kendrick 2003; Christodoulakis and Levine 1987; Christodoulakis and Van Der Ploeg 1987; Leventides and Kollias 2014) to nonlinear (Athanasiou et al. 2008; Athanasiou and Kotsios 2008; Kotsios and Leventidis 2004) and stochastic control applications (Dassios et al. 2014).

The importance of feedback rules for policy design is evident from the fact that for more than 20 years monetary policy decisions have been, to a large extent, based on the Taylor rule (see Taylor 1993); this is a linear feedback policy rule stipulating (in its simplest form) that the interest rate is set based on deviations of inflation and GDP from target levels of inflation and potential GDP, respectively. It is interesting to note here that Taylor presented a rule that had fixed settings for the parameters; in particular:

$\mathrm{r}=\mathrm{p}+0.5 \mathrm{y}+0.5(\mathrm{p}-2)+2$

where $r$ is the federal funds rate, $p$ the rate of inflation and $y$ the percentage deviation of real GDP from a target (e.g., potential output). The rule stipulates that, for example, if GDP exceeds its full-employment level, then nominal interest rates need to be increased.

One of the advantages of adopting the feedback framework is that it allows to explicitly take into account the time lags associated with the conduct of economic policy, since they can be incorporated into the dynamics of the model and the feedback policy rule (Kendrick 1988). Most importantly, the feedback methodology allows for more frequent (and, possibly, smaller) interventions by the policymaker, which are likely to result in a smoother transition path for the economy (see Kendrick and Amman 2014; Kendrick and Shoukry 2014).

Our aim in this paper is to utilize the algorithmic feedback control framework for the design of shortterm fiscal policy interventions. That is, we want to design linear feedback policy rules for the fiscal policy instruments available so that predetermined (fixed) desired sequences for the policy targets (GDP and 
public debt levels) are simultaneously tracked. In particular, we assume that the policymaker has at his disposal two instruments: expenditures related to compensation of public sector employees, social benefits, etc. (i.e., expenditures that cover individual and collective consumption) and expenditures related to investment projects (e.g., infrastructure) that will be funded by the government (either via its own budget or by using external funding such as EU structural funds or the funds available from the so-called Juncker Investment Plan). These investment expenditures are subject to several time lags (including, among others, legislative, design and implementation lags), and as a result, they will affect the economy with a possibly substantial delay; however, the feedback mechanism used allows us to explicitly incorporate these lags into the design of the fiscal policy rules. These rules will provide the exact sequence of the policy instruments necessary to ensure that the target levels of GDP and public debt will be simultaneously met, without any deviation (thus, the tracking error will be equal to zero).

In order to design the policy rules, we use an algorithmic linear feedback control technique known as (exact) model matching control. It is a completely parameterized technique allowing us to develop appropriate symbolic algorithms in order to design the requested policy rules. One of the main advantages of this approach is that we obtain as a solution a class of feedback policy rules; this grants the policymaker the ability to choose the most appropriate policy rule from the set of potential policies available, depending on the particular case at hand. Moreover, the policy rules take into account the state of the economy, since they incorporate the relevant information available up to the decision period, and they are responsive (i.e., the coefficients of the algebraic expressions are not fixed), thus representing a more discretionary approach to the design of fiscal policy.

Our analysis is conducted in the context of a linear, deterministic variant of the standard multiplieraccelerator model proposed by Samuelson (1939). The main reason for choosing this simple linear model is its tractability, as it will allow us to thoroughly examine the effects of the proposed methodology on the workings of the system.

The rest of this paper is organized as follows: Sect. 2 presents the model, in Sect. 3 we develop the proposed methodology, and Sect. 4 contains some simulations and the main results of the paper. Section 5 concludes.

\section{CONCLUSION}

In this paper, we presented an application of algorithmic linear feedback control for the design of shortterm fiscal policy. In particular, in the context of a linear deterministic variant of the multiplier-accelerator model, using an algebraic control theory technique known as exact model matching, we designed a class of linear feedback laws such that the system will immediately track a predetermined, desired trajectory for both policy targets, without any deviations. Moreover, in order to examine the effects of time lags, we run some simulations under different policy response rates. An important implication of the policy experiments is that immediate response allows the government to achieve the policy targets with relatively small policy interventions, compared to cases where there are larger delays in the disbursement of funds.

\section{REFERENCES}

Amman, H., \& Kendrick, D. (2003). Mitigation of the Lucas critique with stochastic control methods. $J$ Econ Dyn Control, 27, 2035-2057.

Astrom, K., \& Wittenmark, B. (1996). Computer-controlled systems: Theory and design (3rd ed.). Prentice Hall, Prentice.

Athanasiou, G., Karafyllis, I., \& Kotsios, S. (2008). Price stabilization using buffer stocks. J Econ Dyn Control, 32, 1212-1235.

Athanasiou, G., \& Kotsios, S. (2008). An algorithmic approach to exchange rate stabilisation. Econ Model, 25, 1246-1260.

Christodoulakis, N., \& Levine, P. (1987). The trade-off between simplicity and optimality in macroeconomic policy design. J Econ Dyn Control, 11, 173-178. 
Christodoulakis, N., \& Van Der Ploeg, F. (1987). Macrodynamic formulation with connecting views of the economy: A synthesis of optimal control and feedback design. Int J Syst Sci, 18, 449-479.

Dalla, E., Karpetis, C., \& Varelas, E. (2016). Modeling investment cycles: A theoretical analysis. Mod Econ, 7, 336-344.

Dalla, E., \& Varelas, E. (2016). Second-order accelerator of investment: the case of discrete time. Intern Rev Econ Educ, 21, 48-60.

Dassios, I., \& Devine, M. (2016). A macroeconomic mathematical model for the national income of a union of countries with interaction and trade. J Econ Struct, 5, 1-15.

Dassios, I., \& Zimbidis, A. (2014). The classical Samuelson's model in a multi-country context under a delayed framework with interaction. Dyn Contin Discrete Impuls Syst Ser B Appl Algorithms, 21, 261-274.

Dassios, I., Zimbidis, A., \& Kontzalis, C. (2014). The delay effect in a stochastic multiplier-accelerator model. J Econ Struct, 3, 7.

Hommes, C. (1995). A reconsideration of hicks' nonlinear trade cycle model. Struct Change Econ Dyn, 6, 4.

Kendrick, D. (1988). Feedback: A new framework for macroeconomic policy (1st ed.). Springer, Netherlands.

Kendrick, D., \& Amman, H. (2014). Quarterly fiscal policy. Econ Voice, 11, 7-12.

Kendrick, D., \& Shoukry, G. (2014). Quarterly fiscal policy experiments with a multiplier-accelerator model. Comput Econ, 44, 269-293.

Kostarakos, I., \& Kotsios, S. (2017). Fiscal policy design in Greece in the aftermath of the crisis: An algorithmic approach. Comput Econ. doi:10.1007/s10614-017-9650-3

Kotsios, S., \& Kostarakos, I. (2016). Controlling national income and public debt via fiscal policy. A model matching algorithmic approach. Vestnik of Saint-Petersburg University. Series 10. Applied Mathematics. Computer Science. Control Processes, (forthcoming).

Kotsios, S., \& Leventidis, J. (2004). A feedback policy for a modified Samuelson-Hicks model. Int J Syst Sci, 35, 331-341.

Leventides, J., \& Kollias, I. (2014). Optimal control indicators for the assessment of the influence of government policy to business cycle shocks. J Dyn Games, 1, 79-104.

Phillips, A. (1954). Stabilisation policy in a closed economy. Econ J, 64, 290-323.

Puu, T. (2007). The Hicksian trade cycle with floor and ceiling dependent on capital stock. J Econ Dyn Control, 31, 575-592.

Samuelson, P. (1939). Interactions between the multiplier analysis and the principle of acceleration. Rev Econ Stat, 21, 75-78.

Taylor, J. (1993). Discretion versus policy rules in practice. Carnegie-Rochester Conf Ser Public Policy, 39, 195-214.

Zheng, M., \& Tian, V-I. (2019). Empowerment across Cultures: How National Culture Affects Structural and Psychological Empowerment and Employee Engagement. Journal of Marketing Development and Competitiveness, 13(3). https://doi.org/10.33423/jmdc.v13i3.2245 


\section{APPENDIX}

TABLE 1

INITIAL CONDITIONS

\begin{tabular}{|l|l|l|l|l|}
\hline Time & $\boldsymbol{Y}$ & $\boldsymbol{B}$ & $\boldsymbol{G}^{\boldsymbol{I}}$ & $\boldsymbol{G}^{\boldsymbol{w}}$ \\
\hline 1 & 120 & 135 & 14.45 & 27 \\
\hline 2 & 112 & 142 & 10.63 & 25 \\
\hline 3 & 105 & 145 & 6.9 & 33.42 \\
\hline 4 & 100 & 150 & 4.1 & 35.1 \\
\hline
\end{tabular}

FIGURE 1

GENERAL GOVERNMENT EXPENDITURE

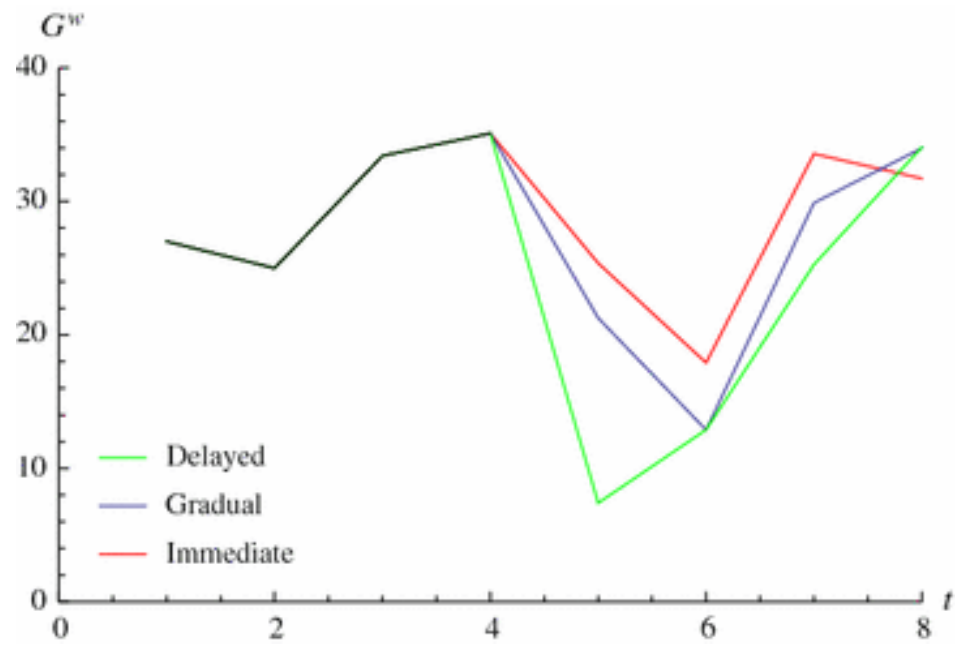

FIGURE 2

GOVERNMENT INVESTMENT EXPENDITURE

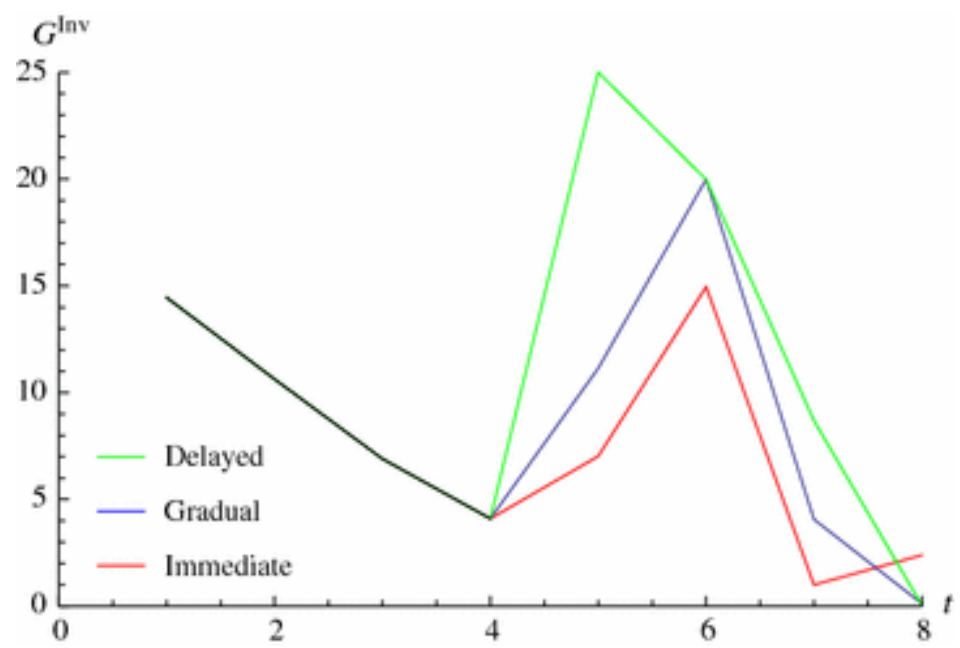




\section{TRANSLATED VERSION: SPANISH}

Below is a rough translation of the insights presented above. This was done to give a general understanding of the ideas presented in the paper. Please excuse any grammatical mistakes and do not hold the original authors responsible for these mistakes.

\section{VERSION TRADUCIDA: ESPAÑOL}

A continuación se muestra una traducción aproximada de las ideas presentadas anteriormente. Esto se hizo para dar una comprensión general de las ideas presentadas en el documento. Por favor, disculpe cualquier error gramatical y no responsabilite a los autores originales de estos errores.

\section{INTRODUCCIÓN}

Uno de los objetivos más importantes de la política económica es garantizar, mediante la manipulación adecuada de los instrumentos de política disponibles (variables de control), que el sistema económico rastree, lo más de cerca posible, el camino deseado para los objetivos de política (productos). Uno de los enfoques que se ha utilizado para el diseño de la política económica es el enfoque de retroalimentación, derivado de la literatura de la teoría del control matemático. Varios aspectos de la metodología de retroalimentación se han utilizado para los fines del diseño de políticas durante más de 50 años, comenzando con el uso de controladores PID en el documento seminal de Phillips (1954). Estos aspectos van desde el control óptimo de la retroalimentación (estocástico) (véase, entre otros, Amman y Kendrick 2003; Christodoulakis y Levine 1987; Christodoulakis y Van Der Ploeg 1987; Leventides y Kollias 2014) a no lineal (Athanasiou et al. 2008; Athanasiou y Kotsios 2008; Kotsios y Leventidis 2004) y aplicaciones de control estocástico (Dassios et al. 2014).

La importancia de las normas de retroalimentación para el diseño de políticas es evidente por el hecho de que durante más de 20 años las decisiones de política monetaria se han basado, en gran medida, en la regla de Taylor (véase Taylor 1993); se trata de una regla de política de retroalimentación lineal que estipula (en su forma más simple) que la tasa de interés se establece en función de las desviaciones de la inflación y el PIB de los niveles objetivo de inflación y el PIB potencial, respectivamente. Es interesante notar aquí que Taylor presentó una regla que tenía configuraciones fijas para los parámetros; en particular:

$\mathrm{r}=\mathrm{p}+0.5 \mathrm{y}+0.5(\mathrm{p}-2)+2$

donde son es la tasa de fondos federales, $\mathrm{p}$ la tasa de inflación e y la desviación porcentual del PIB real de un objetivo (por ejemplo, la producción potencial). La norma estipula que, por ejemplo, si el PIB supera su nivel de pleno empleo, entonces es necesario aumentar las tasas de interés nominales.

Una de las ventajas de adoptar el marco de retroalimentación es que permite tener en cuenta explícitamente los descuidos de tiempo asociados con la conducción de la política económica, ya que pueden incorporarse a la dinámica del modelo y la regla de política de retroalimentación (Kendrick 1988). Lo que es más importante, la metodología de retroalimentación permite intervenciones más frecuentes (y, posiblemente, más pequeñas) por parte de los responsables de la formulación de políticas, lo que probablemente resulte en una trayectoria de transición más suave para la economía (ver Kendrick y Amman 2014; Kendrick y Shoukry 2014).

Nuestro objetivo en este documento es utilizar el marco de control de retroalimentación algorítmica para el diseño de intervenciones de política fiscal a corto plazo. Es decir, queremos diseñar reglas de política de retroalimentación lineal para los instrumentos de política fiscal disponibles, de modo que se realicen simultáneamente un seguimiento de las secuencias deseadas predeterminadas (fijas) para los objetivos de política (PIB y niveles de deuda pública). En particular, suponemos que el responsable político tiene a su disposición dos instrumentos: los gastos relacionados con la remuneración de los empleados del sector público, las prestaciones sociales, etc. (es decir, los gastos que cubren el consumo individual y colectivo) y 
los gastos relacionados con proyectos de inversión (por ejemplo, infraestructuras) que serán financiados por el gobierno (ya sea a través de su propio presupuesto o utilizando fondos externos como los fondos estructurales de la UE o los fondos disponibles del llamado Plan de Inversiones Juncker). Estos gastos de inversión están sujetos a varios retrasos (incluidos, entre otros, retrasos legislativos, de diseño y de aplicación) y, como resultado, afectarán a la economía con un retraso posiblemente sustancial; sin embargo, el mecanismo de retroalimentación utilizado nos permite incorporar explícitamente estos rezags en el diseño de las reglas de política fiscal. Estas normas proporcionarán la secuencia exacta de los instrumentos de política necesarios para garantizar que los niveles objetivo de PIB y deuda pública se cumplan simultáneamente, sin ninguna desviación (por lo tanto, el error de seguimiento será igual a cero).

Para diseñar las reglas de política, utilizamos una técnica algorítmica de control de retroalimentación lineal conocida como control de coincidencia de modelo (exacto). Es una técnica completamente parametrizada que nos permite desarrollar algoritmos simbólicos apropiados para diseñar las reglas de política solicitadas. Una de las principales ventajas de este enfoque es que obtenemos como solución una clase de reglas de política de retroalimentación; esto otorga al responsable de la formulación de políticas la capacidad de elegir la regla de política más apropiada del conjunto de políticas potenciales disponibles, dependiendo del caso particular en cuestión. Además, las normas de política tienen en cuenta el estado de la economía, ya que incorporan la información relevante disponible hasta el período de decisión, y son receptivas (es decir, los coeficientes de las expresiones algebraicas no son fijos), lo que representa un enfoque más discrecional para el diseño de la política fiscal.

Nuestro análisis se lleva a cabo en el contexto de una variante lineal y determinista del modelo estándar multiplicador-acelerador propuesto por Samuelson (1939). La razón principal para elegir este modelo lineal simple es su manejabilidad, ya que nos permitirá examinar a fondo los efectos de la metodología propuesta en el funcionamiento del sistema.

El resto de este trabajo está organizado de la siguiente manera: La Sección 2 presenta el modelo, en la Sección 3 desarrollamos la metodología propuesta, y la Sección 4 contiene algunas simulaciones y los principales resultados del documento. Concluye la sección 5.

\section{CONCLUSIÓN}

En este trabajo, presentamos una aplicación del control algorítmico de retroalimentación lineal para el diseño de la política fiscal a corto plazo. En particular, en el contexto de una variante determinista lineal del modelo multiplicador-acelerador, utilizando una técnica de teoría de control algebraico conocida como coincidencia exacta del modelo, diseñamos una clase de leyes de retroalimentación lineal de tal manera que el sistema rastreará inmediatamente una trayectoria predeterminada y deseada para ambos objetivos de política, sin desviaciones. Además, con el fin de examinar los efectos de los retrasos en el tiempo, realizamos algunas simulaciones bajo diferentes tasas de respuesta política. Una implicación importante de los experimentos de política es que la respuesta inmediata permite al gobierno alcanzar los objetivos de política con intervenciones de política relativamente pequeñas, en comparación con los casos en que hay mayores retrasos en el desembolso de fondos.

\section{TRANSLATED VERSION: FRENCH}

Below is a rough translation of the insights presented above. This was done to give a general understanding of the ideas presented in the paper. Please excuse any grammatical mistakes and do not hold the original authors responsible for these mistakes.

\section{VERSION TRADUITE: FRANÇAIS}

Voici une traduction approximative des idées présentées ci-dessus. Cela a été fait pour donner une compréhension générale des idées présentées dans le document. Veuillez excuser toutes les erreurs grammaticales et ne pas tenir les auteurs originaux responsables de ces erreurs. 


\section{INTRODUCTION}

L'un des objectifs les plus importants de la politique économique est de s'assurer, par la manipulation appropriée des instruments politiques disponibles (variables de contrôle), que le système économique suit, aussi étroitement que possible, une trajectoire souhaitée pour les objectifs politiques (extrants). L'une des approches qui a été utilisée pour la conception de la politique économique est l'approche de rétroaction, découlant de la littérature sur la théorie mathématique du contrôle. Divers aspects de la méthodologie de rétroaction ont été utilisés aux fins de la conception des politiques pendant plus de 50 ans, à commencer par l'utilisation de contrôleurs PID dans l'article fondateur de Phillips (1954). Ces aspects vont du contrôle optimal de la rétroaction (stochastique) (voir, entre autres, Amman et Kendrick 2003; Christodoulakis et Levine, 1987; Christodoulakis et Van Der Ploeg, 1987; Léventide et Kollias, 2014) à non linéaire (Athanasiou et coll., 2008; Athanasiou et Kotsios, 2008; Kotsios et Leventidis 2004) et les applications de contrôle stochastique (Dassios et al. 2014).

L'importance des règles de rétroaction pour la conception des politiques est évidente du fait que, depuis plus de 20 ans, les décisions de politique monétaire sont, dans une large mesure, fondées sur la règle de Taylor (voir Taylor, 1993); il s'agit d'une règle de politique de rétroaction linéaire stipulant (dans sa forme la plus simple) que le taux d'intérêt est fixé en fonction des écarts de l'inflation et du PIB par rapport aux niveaux cibles d'inflation et au PIB potentiel, respectivement. Il est intéressant de noter ici que Taylor a présenté une règle qui avait des paramètres fixes pour les paramètres; en particulier:

$\mathrm{r}=\mathrm{p}+0,5$ ans $+0,5(\mathrm{p}-2)+2$

où sont le taux des fonds fédéraux, $\mathrm{p}$ le taux d'inflation et y l'écart en pourcentage du PIB réel par rapport à une cible (p. ex., la production potentielle). La règle stipule que, par exemple, si le PIB dépasse son niveau de plein emploi, les taux d'intérêt nominaux doivent être augmentés.

L'un des avantages de l'adoption du cadre de rétroaction est qu'il permet de prendre explicitement en compte les décalages temporels associés à la conduite de la politique économique, puisqu'ils peuvent être intégrés dans la dynamique du modèle et la règle de la politique de rétroaction (Kendrick, 1988). Plus important encore, la méthodologie de rétroaction permet des interventions plus fréquentes (et, peut-être, plus petites) de la part des décideurs, ce qui est susceptible d'entraîner une transition plus douce pour l'économie (voir Kendrick et Amman 2014; Kendrick et Shoukry, 2014).

Notre objectif dans cet article est d'utiliser le cadre de contrôle de rétroaction algorithmique pour la conception d'interventions de politique budgétaire à court terme. C'est-à-dire que nous voulons concevoir des règles de politique de rétroaction linéaire pour les instruments de politique budgétaire disponibles afin que les séquences prédéterminées (fixes) souhaitées pour les objectifs politiques (niveaux du PIB et de la dette publique) soient suivies simultanément. En particulier, nous supposons que le décideur politique dispose de deux instruments: les dépenses liées à la rémunération des employés du secteur public, aux prestations sociales, etc. (c'est-à-dire les dépenses qui couvrent la consommation individuelle et collective) et les dépenses liées aux projets d'investissement (par exemple, les infrastructures) qui seront financées par le gouvernement (soit via son propre budget, soit en utilisant des fonds externes tels que les fonds structurels de l'UE ou les fonds disponibles dans le cadre du plan d'investissement Juncker). Ces dépenses d'investissement sont sujettes à plusieurs décalages temporels (y compris, entre autres, des retards législatifs, de conception et de mise en œuvre) et, par conséquent, elles affecteront l'économie avec un retard peut-être important; toutefois, le mécanisme de rétroaction utilisé nous permet d'intégrer explicitement ces retards dans la conception des règles de politique budgétaire. Ces règles fourniront la séquence exacte des instruments de politique nécessaires pour garantir que les niveaux cibles du PIB et de la dette publique seront atteints simultanément, sans aucun écart (ainsi, l'erreur de suivi sera égale à zéro).

Afin de concevoir les règles de stratégie, nous utilisons une technique de contrôle de rétroaction linéaire algorithmique connue sous le nom de contrôle de correspondance de modèle (exact). Il s'agit d'une technique entièrement paramétrée nous permettant de développer des algorithmes symboliques appropriés afin de concevoir les règles de politique demandées. L'un des principaux avantages de cette approche est 
que nous obtenons comme solution une classe de règles de politique de rétroaction; cela donne au décideur la possibilité de choisir la règle politique la plus appropriée parmi l'ensemble des politiques potentielles disponibles, en fonction du cas particulier en question. En outre, les règles de politique tiennent compte de l'état de l'économie, puisqu'elles intègrent les informations pertinentes disponibles jusqu'à la période de décision et qu'elles sont réactives (c'est-à-dire que les coefficients des expressions algébriques ne sont pas fixes), ce qui représente une approche plus discrétionnaire de la conception de la politique budgétaire.

Notre analyse est menée dans le contexte d'une variante linéaire et déterministe du modèle standard multiplicateur-accélérateur proposé par Samuelson (1939). La principale raison du choix de ce modèle linéaire simple est sa tractabilité, car il nous permettra d'examiner en profondeur les effets de la méthodologie proposée sur le fonctionnement du système.

Le reste de cet article est organisé comme suit: la section 2 présente le modèle, dans la section 3 , nous développons la méthodologie proposée, et la section 4 contient quelques simulations et les principaux résultats de l'article. La section 5 se termine.

\section{CONCLUSION}

Dans cet article, nous avons présenté une application du contrôle de rétroaction linéaire algorithmique pour la conception de la politique budgétaire à court terme. En particulier, dans le contexte d'une variante déterministe linéaire du modèle multiplicateur-accélérateur, en utilisant une technique de théorie algébrique du contrôle connue sous le nom d'appariement exact du modèle, nous avons conçu une classe de lois de rétroaction linéaires de sorte que le système suivra immédiatement une trajectoire prédéterminée et souhaitée pour les deux cibles politiques, sans aucun écart. De plus, afin d'examiner les effets des décalages temporels, nous faisons des simulations selon différents taux de réponse des politiques. Une implication importante des expériences politiques est que la réponse immédiate permet au gouvernement d'atteindre les objectifs politiques avec des interventions politiques relativement petites, par rapport aux cas où il y a des retards plus importants dans le décaissement des fonds.

\section{TRANSLATED VERSION: GERMAN}

Below is a rough translation of the insights presented above. This was done to give a general understanding of the ideas presented in the paper. Please excuse any grammatical mistakes and do not hold the original authors responsible for these mistakes.

\section{ÜBERSETZTE VERSION: DEUTSCH}

Hier ist eine ungefähre Übersetzung der oben vorgestellten Ideen. Dies wurde getan, um ein allgemeines Verständnis der in dem Dokument vorgestellten Ideen zu vermitteln. Bitte entschuldigen Sie alle grammatikalischen Fehler und machen Sie die ursprünglichen Autoren nicht für diese Fehler verantwortlich.

\section{EINLEITUNG}

Eines der wichtigsten Ziele der Wirtschaftspolitik ist es, durch die entsprechende Manipulation der verfügbaren politischen Instrumente (Kontrollvariablen) sicherzustellen, dass das Wirtschaftssystem einen gewünschten Weg für die politischen Ziele (Outputs) so genau wie möglich verfolgt. Einer der Ansätze, die für die Gestaltung der Wirtschaftspolitik verwendet wurden, ist der Feedback-Ansatz, der aus der mathematischen Regeltheorieliteratur stammt. Verschiedene Aspekte der Feedback-Methodik werden seit mehr als 50 Jahren für die Zwecke der Politikgestaltung verwendet, beginnend mit der Verwendung von PID-Controllern in der bahnbrechenden Arbeit von Phillips (1954). Diese Aspekte reichen von der (stochastischen) optimalen Rückkopplungskontrolle (siehe u.a. Amman und Kendrick 2003; Christodoulakis und Levine 1987; Christodoulakis und Van Der Ploeg 1987; Leventides und Kollias 2014) 
bis nichtlinear (Athanasiou et al. 2008; Athanasiou und Kotsios 2008; Kotsios und Leventidis 2004) und stochastische Kontrollanwendungen (Dassios et al. 2014).

Die Bedeutung von Feedback-Regeln für die Politikgestaltung zeigt sich daran, dass geldpolitische Entscheidungen seit mehr als 20 Jahren weitgehend auf der Taylor-Regel basieren (siehe Taylor 1993); Dies ist eine lineare Regel der Rückkopplungspolitik, die (in ihrer einfachsten Form) festlegt, dass der Zinssatz auf der Grundlage von Abweichungen der Inflation und des BIP von den Zielniveaus der Inflation bzw. des potenziellen BIP festgelegt wird. Es ist interessant festzustellen, dass Taylor eine Regel vorgestellt hat, die feste Einstellungen für die Parameter hatte; besonders:

$\mathrm{r}=\mathrm{p}+0,5 \mathrm{y}+0,5(\mathrm{p}-2)+2$

wobei die Federal Funds Rate, $\mathrm{p}$ die Inflationsrate und y die prozentuale Abweichung des realen BIP von einem Ziel (z. B. Produktionspotenzial) ist. Die Regel sieht vor, dass beispielsweise, wenn das BIP sein Vollbeschäftigungsniveau übersteigt, die Nominalzinsen erhöht werden müssen.

Einer der Vorteile der Übernahme des Feedback-Frameworks besteht darin, dass es ermöglicht, die mit der Durchführung der Wirtschaftspolitik verbundenen Zeitverzögerungen explizit zu berücksichtigen, da sie in die Dynamik des Modells und der Feedback-Policy-Regel einbezogen werden können (Kendrick 1988). Am wichtigsten ist, dass die Feedback-Methodik häufigere (und möglicherweise kleinere) Interventionen des politischen Entscheidungsträgers ermöglicht, die wahrscheinlich zu einem reibungsloseren Übergangspfad für die Wirtschaft führen werden (siehe Kendrick und Amman 2014; Kendrick und Shoukry 2014).

Unser Ziel in diesem Artikel ist es, das algorithmische Feedback-Kontroll-Framework für die Gestaltung kurzfristiger fiskalpolitischer Interventionen zu nutzen. Das heißt, wir wollen lineare FeedbackPolicy-Regeln für die verfügbaren fiskalpolitischen Instrumente entwerfen, so dass vorgegebene (feste) Wunschsequenzen für die policy targets (BIP und Public Debt Levels) gleichzeitig nachverfolgt werden. Insbesondere gehen wir davon aus, dass der politische Entscheidungsträger über zwei Instrumente verfügt: Ausgaben im Zusammenhang mit der Vergütung von Beschäftigten des öffentlichen Sektors, Sozialleistungen usw. (d. h. Ausgaben, die den individuellen und kollektiven Konsum decken) und Ausgaben im Zusammenhang mit Investitionsprojekten (z. B. Infrastruktur), die von der Regierung finanziert werden (entweder über ihren eigenen Haushalt oder durch die Verwendung externer Mittel wie EU-Strukturfonds oder der aus der sogenannten Juncker-Investitionsoffensive verfügbaren Mittel). Diese Investitionsausgaben unterliegen mehreren zeitlichen Verzögerungen (einschließlich unter anderem Gesetzgebungs-, Design- und Umsetzungsverzögerungen), und infolgedessen werden sie die Wirtschaft mit einer möglicherweise erheblichen Verzögerung beeinträchtigen; Der verwendete Feedback-Mechanismus ermöglicht es uns jedoch, diese Verzögerungen explizit in die Gestaltung der finanzpolitischen Regeln einzubeziehen. Diese Regeln werden die genaue Reihenfolge der politischen Instrumente vorsehen, die erforderlich sind, um sicherzustellen, dass die Zielniveaus des BIP und der öffentlichen Verschuldung gleichzeitig ohne Abweichung erreicht werden (daher ist der Tracking Error gleich Null).

Um die Richtlinienregeln zu entwerfen, verwenden wir eine algorithmische lineare FeedbackSteuerungstechnik, die als (exakte) Modellabgleichssteuerung bekannt ist. Es ist eine vollständig parametrisierte Technik, die es uns ermöglicht, geeignete symbolische Algorithmen zu entwickeln, um die angeforderten Richtlinienregeln zu entwerfen. Einer der Hauptvorteile dieses Ansatzes besteht darin, dass wir als Lösung eine Klasse von Feedback-Richtlinienregeln erhalten. Dies gibt dem politischen Entscheidungsträger die Möglichkeit, je nach Fall die am besten geeignete politische Regel aus den verfügbaren potenziellen Politiken auszuwählen. Darüber hinaus berücksichtigen die politischen Regeln den Zustand der Wirtschaft, da sie die relevanten Informationen enthalten, die bis zum Entscheidungszeitraum verfügbar sind, und sie sind reaktionsschnell (d. h. die Koeffizienten der algebraischen Ausdrücke sind nicht festgelegt), was einen diskretionäreren Ansatz für die Gestaltung der Fiskalpolitik darstellt.

Unsere Analyse wird im Kontext einer linearen, deterministischen Variante des von Samuelson (1939) vorgeschlagenen Standard-Multiplikator-Beschleuniger-Modells durchgeführt. Der Hauptgrund für die 
Wahl dieses einfachen linearen Modells ist seine Traktionsfähigkeit, da es uns ermöglicht, die Auswirkungen der vorgeschlagenen Methodik auf die Funktionsweise des Systems gründlich zu untersuchen.

Der Rest dieses Papiers ist wie folgt organisiert: Abschnitt 2 stellt das Modell vor, in Abschnitt 3 entwickeln wir die vorgeschlagene Methodik und Abschnitt 4 enthält einige Simulationen und die wichtigsten Ergebnisse des Papiers. Abschnitt 5 schließt ab.

\section{SCHLUSSFOLGERUNG}

In diesem Artikel haben wir eine Anwendung der algorithmischen linearen Rückkopplungskontrolle für die Gestaltung der kurzfristigen Fiskalpolitik vorgestellt. Insbesondere im Kontext einer linearen deterministischen Variante des Multiplikator-Beschleuniger-Modells haben wir unter Verwendung einer algebraischen Kontrolltheorietechnik, die als exaktes Modellabgleich bekannt ist, eine Klasse linearer Rückkopplungsgesetze entworfen, so dass das System sofort eine vorgegebene, gewünschte Trajektorie für beide politischen Ziele ohne Abweichungen verfolgt. Um die Auswirkungen von Zeitverzögerungen zu untersuchen, führen wir außerdem einige Simulationen unter verschiedenen politischen Antwortraten durch. Eine wichtige Implikation der politischen Experimente ist, dass die sofortige Reaktion es der Regierung ermöglicht, die politischen Ziele mit relativ kleinen politischen Interventionen zu erreichen, verglichen mit Fällen, in denen es größere Verzögerungen bei der Auszahlung von Mitteln gibt.

\section{TRANSLATED VERSION: PORTUGUESE}

Below is a rough translation of the insights presented above. This was done to give a general understanding of the ideas presented in the paper. Please excuse any grammatical mistakes and do not hold the original authors responsible for these mistakes.

\section{VERSÃO TRADUZIDA: PORTUGUÊS}

Aqui está uma tradução aproximada das ideias acima apresentadas. Isto foi feito para dar uma compreensão geral das ideias apresentadas no documento. Por favor, desculpe todos os erros gramaticais e não responsacule os autores originais responsáveis por estes erros.

\section{INTRODUÇÃO}

Um dos objetivos mais importantes da política econômica é garantir, através da adequada manipulação dos instrumentos políticos disponíveis (variáveis de controle), que o sistema econômico trilha, o mais de perto possível, um caminho desejado para as metas políticas (saídas). Uma das abordagens utilizadas para o desenho da política econômica é a abordagem do feedback, decorrente da literatura teoria do controle matemático. Vários aspectos da metodologia de feedback têm sido utilizados para fins de design de políticas há mais de 50 anos, começando com o uso de controladores de PID no papel seminal por Phillips (1954). Esses aspectos variam de controle de feedback ideal (estocástico) (ver, entre outros, Amã e Kendrick 2003; Christodoulakis e Levine 1987; Christodoulakis e Van Der Ploeg 1987; Leventides e Kollias 2014) para não linear (Athanasiou et al. 2008; Athanasiou e Kotsios 2008; Kotsios e Leventidis 2004) e aplicações de controle estocástico (Dassios et al. 2014).

A importância das regras de feedback para o desenho de políticas é evidente pelo fato de que há mais de 20 anos as decisões de política monetária têm sido, em grande parte, baseadas na regra de Taylor (ver Taylor 1993); trata-se de uma regra de política de feedback linear estipulando (em sua forma mais simples) que a taxa de juros é definida com base em desvios da inflação e do PIB dos níveis-alvo da inflação e do PIB potencial, respectivamente. É interessante notar aqui que Taylor apresentou uma regra que tinha configurações fixas para os parâmetros; em particular: 
onde estão a taxa de fundos federais, $\mathrm{p}$ a taxa de inflação e y o desvio percentual do PIB real de uma meta (por exemplo, produção potencial). A regra estipula que, por exemplo, se o PIB excede seu nível de pleno emprego, então as taxas de juros nominais precisam ser aumentadas.

Uma das vantagens de adotar o quadro de feedback é que ele permite levar em conta explicitamente os atrasos de tempo associados à condução da política econômica, uma vez que podem ser incorporados à dinâmica do modelo e à regra da política de feedback (Kendrick 1988). Mais importante, a metodologia de feedback permite intervenções mais frequentes (e, possivelmente, menores) por parte do formulador de políticas, que provavelmente resultarão em um caminho de transição mais suave para a economia (ver Kendrick e Amã 2014; Kendrick e Shoukry 2014).

Nosso objetivo neste artigo é utilizar a estrutura de controle de feedback algorítmico para o desenho de intervenções de política fiscal de curto prazo. Ou seja, queremos desenhar regras de política de feedback linear para os instrumentos de política fiscal disponíveis para que as sequências pré-determinadas (fixas) desejadas para as metas políticas (PIB e níveis de dívida pública) sejam simultaneamente rastreadas. Em particular, assumimos que o formulador de políticas tem à sua disposição dois instrumentos: despesas relacionadas à remuneração dos funcionários do setor público, benefícios sociais, etc. (ou seja, despesas que cobrem o consumo individual e coletivo) e despesas relacionadas a projetos de investimento (por exemplo, infraestrutura) que serão financiadas pelo governo (seja através de seu próprio orçamento ou usando fundos externos, como fundos estruturais da UE ou os fundos disponíveis do chamado Plano de Investimento Juncker). Esses gastos com investimentos estão sujeitos a várias defasagens de tempo (incluindo, entre outros, atrasos legislativos, de projeto e implementação), e, como resultado, afetarão a economia com um atraso possivelmente substancial; no entanto, o mecanismo de feedback utilizado nos permite incorporar explicitamente esses atrasos no desenho das regras da política fiscal. Essas regras fornecerão a sequência exata dos instrumentos políticos necessários para garantir que os níveis-alvo do PIB e da dívida pública sejam simultaneamente cumpridos, sem qualquer desvio (portanto, o erro de rastreamento será igual a zero).

Para projetar as regras da política, usamos uma técnica de controle de feedback linear algorítmico conhecida como controle de correspondência de modelos (exatos). É uma técnica completamente parametrizada que nos permite desenvolver algoritmos simbólicos apropriados para projetar as regras de política solicitadas. Uma das principais vantagens dessa abordagem é que obtemos como solução uma classe de regras de política de feedback; isso confere ao formulador de políticas a capacidade de escolher a regra política mais adequada a partir do conjunto de políticas potenciais disponíveis, dependendo do caso específico em questão. Além disso, as regras políticas levam em conta o estado da economia, uma vez que incorporam as informações relevantes disponíveis até o período de decisão, e respondem (ou seja, os coeficientes das expressões algébridas não são fixados), representando assim uma abordagem mais discricionária para a concepção da política fiscal.

Nossa análise é conduzida no contexto de uma variante linear e determinística do modelo multiplicadoracelerador padrão proposto por Samuelson (1939). A principal razão para escolher este modelo linear simples é sua trato, pois nos permitirá examinar minuciosamente os efeitos da metodologia proposta sobre o funcionamento do sistema.

O restante deste artigo é organizado da seguinte forma: A Seita 2 apresenta o modelo, na Seita 3 desenvolvemos a metodologia proposta, e a Seita 4 contém algumas simulações e os principais resultados do artigo. A seção 5 termina.

\section{CONCLUSÃO}

Neste artigo, apresentamos uma aplicação de controle de feedback linear algorítmico para o desenho da política fiscal de curto prazo. Em particular, no contexto de uma variante determinística linear do modelo multiplicador-acelerador, usando uma técnica de teoria do controle algébrico conhecida como correspondência exata de modelos, projetamos uma classe de leis de feedback linear, de tal forma que o 
sistema rastreará imediatamente uma trajetória pré-determinada e desejada para ambos os alvos políticos, sem desvios. Além disso, para examinar os efeitos dos atrasos de tempo, executamos algumas simulações sob diferentes taxas de resposta política. Uma implicação importante dos experimentos políticos é que a resposta imediata permite que o governo atinja as metas políticas com intervenções políticas relativamente pequenas, em comparação com os casos em que há maiores atrasos no desembolso de fundos. 